\title{
Understanding Social Machines
}

\author{
Ramine Tinati, Leslie Carr \\ Electronics and Computer Science \\ University of Southampton \\ Southampton, United Kingdom \\ \{rt506, lac\}@ecs.soton.ac.uk
}

\section{Keywords-Social Machines; Social Theory; Methodology; Framework; Web Science}

\section{EXTENDED ABSTRACT}

The Web is an evolving, complex socio-technical system [1]. As a social technology [2], the Web is a diverse collection of interacting networks of activities and communities. The openness and scalability of the Web has provided a platform for new technologies, services and standards, enabling individuals at scale to connect, communicate and share in ways that were previously impractical. The Web has enabled new forms of activity to emerge and grow; a new area of Web activity is the development of social computing and social machines [3]. This new area of activity harnesses the power of the crowd to perform computationally difficult and time consuming tasks.

Before Web technologies provided a platform to perform such tasks, the Internet offered a network of distributed computing power to perform computationally intense tasks [4]. Unlike Web based systems, the design of Internet based systems did not require individuals to directly spend their time operating them, yet they were still a social machine of sorts. Users were required to actively consent to download and install software and use their idle CPU and network bandwidth. The success of these social machines were the result of the heterogeneous networks of human and technologies interacting and working together; these machines relied no only on the technology working, but the acceptance and adoption of a new social process by the individual. It becomes clear that examining the relationship between society and technology, any task that requires the co-constitutional involvement of humans and technologies is a form of social machine, and to this ends, no more so than the Web itself. There just exist different scales of social machinery.

Examining the scales of social machines, they range from the lightweight to heavyweight [5]. Those that are lightweight could be described as exhibiting strict, deterministic characteristics, harnessing the collective problem solving capabilities of humans to perform computationally hard processes [6][7]. Less deterministic are middleweight social machines, exhibiting a mix of lightweight and heavyweight characteristics [8]; lightweight in regards to the crowdsource techniques employed, yet heavyweight in terms of the pre-exiting structures and hierarchy of power that ultimately, make the final decisions. At the heavyweight end of the scale are machines that exist as a result of the interactions between human creativity and technological capabilities, a relationship which is mutually reinforcing [3], power between the user and engineer is distributed, and users are crucial in the machines success and shape the overall goals and purpose of the system [5].

A common characteristic that is shared amongst social machines of all scales is their socio-technical structure, a network of actors that relies on the co-constructive relationships between society and technology [1]. As these machines grow and evolve, they enroll more users, more technologies, and consequently, more issues.

The development of a social machines is in some respects a reflection of the process of the Web [9]; an idea is developed in a controlled environment, and although is subject to the same socio-technical processes of any technological development, the actors involved within this stage are well defined and somewhat restricted. However, when the shift from the micro to the macro occurs-from the controlled environment to the unknowns of the Web-the original actors are no longer in control; consequently the social machine becomes just another Web activity. Therein lies the problem, we currently have no methods to understand and describe the social machines during and after its development; this not only at the micro level-which itself is important-but at the macro level, when it enters the wilderness of the Web, a Web full of unknowns, competing technologies, diverse users and constantly changing networks of activity.

To address this, we have developed a novel approach to understanding and guiding the development of social machines. The framework, shown in Fig. 1, combines an applied social theory with a mixed methods approach, which takes advantage of not only the richness and complementary analytical strengths that using both quantitative and qualitative data sources provide [10], but also the epistemological and ontological position of Actor Network Theory (ANT), offering an analytical lens to interpret and understanding how socio-technical networks function. ANT provides the language and ontological concepts to explore the structure and formation of the Web activity being 


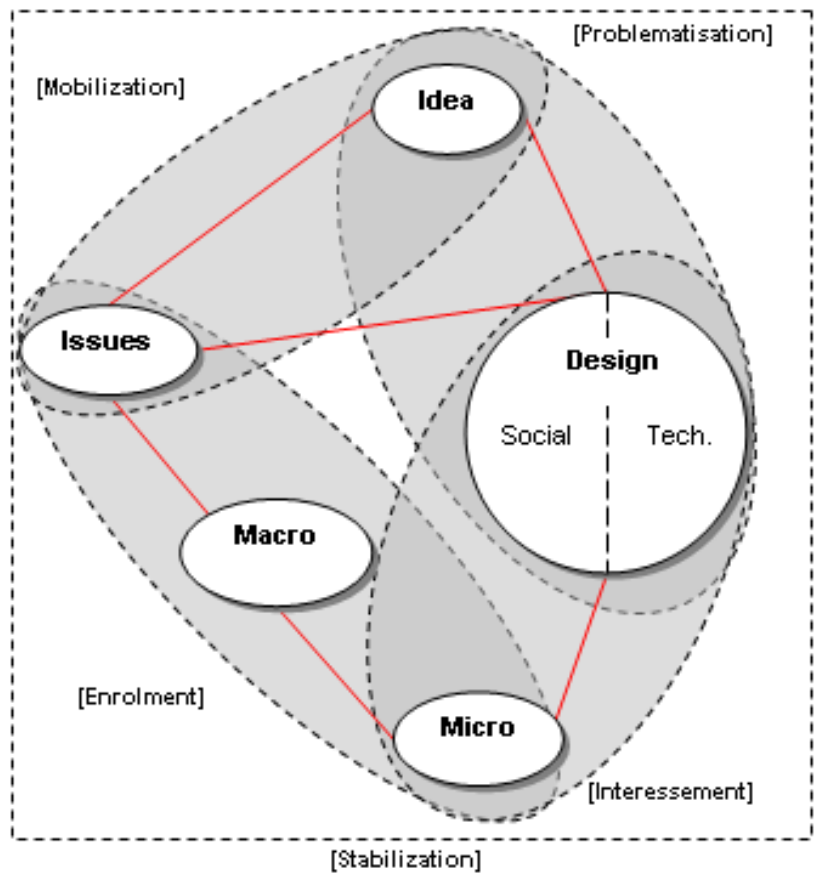

Figure 1. The Process of the Web

studied, based upon ANT's perspective on the formation of actor-networks, their transient characteristics, and their ability to grow, change shape, and join with other networks [11].

Drawing upon a mixed methods approach, the development of a social machine can be examined in terms of the different stages of translation it goes through, examining how the socio-technical activities of the actors enabled the network to translate and reach a potential point of stability. By using the process of translation as a framework to identify and separate a number of different processes required for the successful formation of a network, it provides a method to apply metrics to proactively and reactively understand and support the network's success.

At each stage of translation, a number of data collection techniques which are based on quantitative and/or qualitative data sources can be used, providing evidence based explanations to the interactions and outcomes that result. The framework facilitates a process what might be described by analogy as "social machine engineering".

\section{CONCLUDING REMARKS}

This framework introduced in this paper aims to reflect the characteristics that social machines have been described to have. The framework uses a mixed methods approach underpinned by social theory to provide a detailed and rich understanding of the socio-technical nature of a social machine. The strength of this lies in the diversity of the data being used; whilst the quantitative approach can provide mathematical rigor to the structure and properties of the networks and appreciate its scale, the qualitative approach seeks to examine the 'social relations' [12], and the context to how the social machine is enabling humans and technologies to interact and shape each other. Like many studies using empirical-based research [10], this framework takes advantage of the complementary nature that mixed methods offers, and pushes it further by using an analytical socio-technical lens.

\section{ACKNOWLEDGEMENTS}

This research was funded by the Research Councils UK Digital Economy Programme, Web Science Doctoral Training Centre, EP/G036926/1.

\section{REFERENCES}

[1] S. Halford, C. Pope, and L. Carr, "A Manifesto for Web Science?," Proceedings of the Web Science 2009, pp. 1-6, 2009.

T. Berners-Lee, D. J. Weitzner, W. Hall, K. O'Hara, N. Shadbolt, and J. a. Hendler, "A Framework for Web Science," Foundations and Trends® in Web Science, vol. 1, no. 1, pp. 1-130, 2006.

[3] J. Hendler and T. Berners-Lee, "From the Semantic Web to social machines: A research challenge for AI on the World Wide Web," Artificial Intelligence, vol. 174, no. 2, pp. 156-161, Feb. 2010.

[4] S. M. Larson, C. D. Snow, M. Shirts, and V. S. Pande, "Folding@Home and Genome@Home: Using distributed computing to tackle previously intractable problems in computational biology," Security, 2009.

[5] C. Haythornthwaite, "Crowds and Communities : Light and Heavyweight Models of Peer Production," pp. 1-10, 2009.

[6] W. Roush, "Social Machines: Computing means connecting," Technology Review Published by MIT, vol. 2005, no. 3 August, 2005.

[7] F. Guinchiglia and D. Robertson, "The Social Computer Combining Machine and Human," Computer, no. May, 2010.

[8] M. J. Raddick et al., "Galaxy Zoo: Exploring the Motivations of Citizen Science Volunteers," Astronomy Education Review, vol. 9, no. 1, p. 010103, 2010.

[9] T. Berners-Lee, "The Two Magics of Web Science," in Keynote Speech at 16th International World Wide Web Conference (WWW2007), 2007.

[10] J. E. M. Sale and K. Brazil, "Revisiting the QuantitativeQualitative Debate : Implications for Mixed-Methods Research," Community Health, pp. 43-53, 2002.

[11] B. Latour, Reassembling the Social: An Introduction to Actor-Network-Theory by Bruno Latour, vol. 10, no. 3. Oxford University Press, 2005.

[12] R. S. Burt, "Applied Network Analysis: An Overview," Sociological Methods Research, vol. 7, no. 2, pp. 123130, 1978. 